\title{
ON RESTRICTIONS OF OPERATORS
}

\author{
JOHN WERMER ${ }^{1}$
}

Introduction. Let $\tau$ be a bounded linear operator on a Banach space $B$ and let $\tau^{-1}$ exist. Consider a closed subspace $C$ of $B$ which is invariant under $\tau$, i.e. $\tau C \subseteq C$. The restriction of $\tau$ to $C$ is an operator $T$ on the Banach space $C$. Suppose now that $\tau^{-1} C \Phi C$, so that $T$ has no inverse. The class of operators $T$ arising in this way has rather special properties, which are in general quite different from those of $\tau$ on the whole space $B$. The purpose of this paper is to study this class of restriction operators $T$.

Throughout we shall make one simplifying assumption on $C$, namely, that $C$ contains a single "generator" $x$, i.e. a vector $x$ such that the vectors $T^{n} x, n=0,1,2, \cdots$, are fundamental in $C$. It is clear that if $\tau^{-1} C \Phi C$ and $x$ is a generator of $C$, then $\tau^{-1} x \notin C$.

In order that for a given operator $\tau$ there may exist an invariant subspace $C$ which is not invariant under $\tau^{-1}$, it is necessary that the resolvent set of $\tau$ have more than one component. For let $x$ be in $C$ and take any functional $\psi$ with $\psi(C)=0$. Then $\psi\left((\lambda I-\tau)^{-1} x\right)$ $=\sum_{0}^{\infty}\left(\tau^{n} x, \psi\right) / \lambda^{n+1}=0$ for large $|\lambda|$ and hence for the entire resolvent set if this set is connected. In particular, $\psi\left(\tau^{-1} x\right)=0$; thus $\psi(C)=0$ implies $\psi\left(\tau^{-1} x\right)=0$ and so $\tau^{-1} x \in C$. Hence $\tau^{-1} C \subseteq C$.

In $\$ 1$ we shall show how an operator $T$ on $C$, obtained by restricting $\tau$ to $C$, can be represented as an operator of multiplication by $z$ on a space of analytic functions. We shall also investigate the ring of operators on $C$ which commute with $T$. In $\$ 2$ we shall show how these general considerations specialise in the case of certain normal operators on Hilbert space. In particular, we shall discuss some results of Kolmogoroff on stationary sequences which correspond in our problem to the case of a unitary operator $\tau$ on Hilbert space.

1. Throughout the following we mean by $C$ a closed subspace invariant under $\tau$, with $\tau^{-1} C \Phi C$, and such that $C$ has a generator $x$. $T$ denotes the restriction of $\tau$ to $C$. By $\Phi$ we mean the component of the resolvent set of $\tau$ which includes the origin. $C^{n}, n \geqq 1$, denotes the range of $T^{n}$ and $C^{\infty}$ the intersection of all the $C^{n}$. Each $C^{n}$ is a closed subspace of $C$, for let $y_{k}$ be in $C^{n}, \lim _{k=\infty} y_{k}=y$. We have $y_{k}=T^{n} y_{k}{ }^{\prime}$ where $y_{k}^{\prime} \in C$. Hence $\tau^{-n} y=\lim y_{k}^{\prime} \in C$, since all $y_{k}^{\prime} \in C$, and so $y \in C^{n}$. 1953.

Presented to the Society, February 28, 1953; received by the editors February 26,

1 The author is a Fellow of the National Science Foundation. 
It follows that $C^{\infty}$ is a closed subspace of $C$, since $C^{\infty}$ is the intersection of all $C^{n}$.

Lemma 1. For each $f$ in $C$ there is a unique sequence of numbers $f_{n}, n=0,1, \cdots$, such that for each $N, f-\sum_{n=0}^{N} f_{n} T^{n} x \in C^{N+1}$.

Proof. Consider the numerical functional $\psi_{0}$ which gives to each vector in $C$ of the form $P(T) x$, where $P$ is a polynomial, the value $P(0)$. Now $x \notin C^{1}$ since $\tau^{-1} x \notin C$, and so $x$ has a positive distance $\delta$ from $C^{1}$. If $P$ is any polynomial with $P(0) \neq 0$, then $\|P(T) x / P(0)\| \geqq \delta$, and so $|P(0)| \leqq\|P(T) x\| / \delta$. Thus $\psi_{0}$ is a bounded linear functional on a dense subset of $C$ and so may be extended to be defined and continuous on all of $C$, assigning to each $f$ in $C$ a value $f_{0}$. If now $f \in C$, we have polynomials $P_{n}$ with $\lim _{n=\infty} P_{n}(T) x=f$. Hence $f-f_{0} x=\lim _{n=\infty}\left(P_{n}(T) x-P_{n}(0) x\right)$. But each $P_{n}(T) x-P_{n}(0) x \in C^{1}$ and so $f-f_{0} x \in C^{1}$. Now the same argument we just used for any $f$ in $C$ may be used for any $g$ in $C^{1}$ to give $g-g_{1} T x$ in $C^{2}$ and so for any $f$ in $C$, we have numbers $f_{0}, f_{1}$ with $f-f_{0} x-f_{1} T x \in C^{2}$. We can clearly continue this process up to any $n$. It only remains to show that if $f-\sum_{0}^{n-1} f_{i} T^{i} x \in C^{n}$ and $f-\sum_{0}^{n-1} f_{i}^{\prime} T^{i} x \in C^{n}$, then $f_{i}=f_{i}^{\prime}$, $i=0,1, \cdots, n-1$. But $f_{0}=f_{0}^{\prime}$, for else $x \in C^{1}$, and in the same way $f_{1}=f_{1}^{\prime}$ and so on.

THEOREM 1. Let $\psi$ be any bounded linear functional on $B$ which annihilates $C$ and with $\psi\left(\tau^{-1} x\right) \neq 0$. Let $R_{z}=(z-\tau)^{-1}$. If now $f \in C$ and $\left\{f_{n}\right\}_{0}^{\infty}$ is the sequence associated to $f$ by Lemma 1 , then

$$
\sum_{0}^{\infty} f_{n} z^{n}=\frac{\left(R_{z} f, \psi\right)}{\left(R_{z} x, \psi\right)}
$$

in some circle $|z|<r$ and the right-hand side is analytic in $\mathcal{D}$.

Proof. Let $\psi$ be as described and let $P(T)$ be any polynomial in $T$. Then $R_{z}(P(z)-P(T))$ is again a polynomial in $T$ and so $\left(R_{z}(P(T)\right.$ $-P(z)) x, \psi)=0$. Hence

$$
P(z)=\frac{\left(R_{z} P(T) x, \psi\right)}{\left(R_{z} x, \psi\right)}
$$

and the denominator $\left(R_{z} x, \psi\right) \neq 0$ except at isolated points in $\mathcal{D}$, since it is analytic in $\Phi$ and at 0 equals $\left(\tau^{-1} x, \psi\right) \neq 0$.

Let now $f$ be in $C$. Then we can find a sequence of polynomials $P_{n}$ with $f=\lim _{n=\infty} P_{n}(T) x$. Fix $z_{0}$ in $\mathscr{D}$. Choose now a simple closed contour $\gamma$ contained with its interior in $\Phi$ and containing in its interior the origin and the point $z_{0}$, and such that $\left(R_{2} x, \psi\right) \neq 0$ on $\gamma$. Then 
there exists $\delta>0, M<\infty$, with $\left|\left(R_{x} x, \psi\right)\right| \geqq \delta$ on $\gamma$ and $\left\|R_{\varepsilon}\right\| \leqq M$ on $\gamma$. Hence, by (2), $\left|P_{n}(z)-P_{m}(z)\right| \leqq(M / \delta)\left\|P_{n}(T) x-P_{m}(T) x\right\| \cdot\|\psi\|$ for all $z$ on $\gamma$, and so by the maximum principle for all $z$ in the interior of $\gamma$. Also clearly $\lim _{n, m-\infty}\left\|P_{n}(T) x-P_{m}(T) x\right\|=0$ and so $\lim _{n, m-\infty} \mid P_{n}(z)$ $-P_{m}(z) \mid=0$, whence the sequence $P_{n}(z)$ converges in the interior of $\gamma$ to a function $F(z)$ analytic inside $\gamma$. But now $P_{n}(T) x$ converges to $f$ and so, by (2), $F(z)=\left(R_{z} f, \psi\right) /\left(R_{z} x, \psi\right)$ for $z$ inside $\gamma$. Thus we see that $\left(R_{a} f, \psi\right) /\left(R_{x} x, \psi\right)$ is analytic at every point $z_{0}$ in $\mathscr{D}$. Also we see that if $P_{n}(T) x$ and $Q_{n}(T) x$ converge to $f$, then $\lim _{n-\infty} P_{n}(z)=\lim _{n-\infty} Q_{n}(z)$ for all $z$ in $D$.

Let now $\psi_{n}$ be the functional on $C$ with $\psi_{n}(f)=f_{n}$, for all $f$ in $C$. By the construction of the $f_{n}, \psi_{n}$ is bounded. Choose polynomials $P_{k}$ with $P_{k}(T) x$ converging to $f$. Then

$$
f_{n}=\psi_{n}(f)=\lim _{k \rightarrow \infty} \psi_{n}\left(P_{k}(T) x\right)=\lim _{k \rightarrow \infty} \frac{1}{n !} P_{k}^{(n)}(0) .
$$

Set $F(z)=\left(R_{z} f, \psi\right) /\left(R_{z} x, \psi\right)$. By the above, $F(z)=\lim _{k-\infty} P_{k}(z)$ uniformly in some circle $|z|<r$. Hence $f_{n}=(1 / n !) F^{(n)}(0)$ and so $F(z)$ $=\sum_{0}^{\infty} f_{n} z^{n}$, as asserted.

Note. Formula (2) was suggested to the author by an interpolation formula for polynomials given by Hall in $[1$, p. 32].

THEOREM 2. The mapping: $f$ into $F(z)=\sum_{0}^{\infty} f_{n} z^{n}$ is a homomorphism of $C$ onto a space $\mathcal{F}$ of functions analytic in $\mathcal{D}$, and $T$ is represented on $\mathcal{F}$ as multiplication by $z$. The kernel of the homomorphism is $C^{\infty}$. If $f^{(n)}$ converges to $f$ in $C, F^{(n)}$ converges to $F$ uniformly on every compact subset of $\Phi$.

Proof. It is clear that the mapping is linear. Since $(T f)_{n}=f_{n-1}, T f$ maps into $z F(z)$. If for a certain $f$ in $C, F(z) \equiv 0$, then $f_{n}=0$ for each $n$ and so $f \in C^{n}$ for every $n$. Thus $f \in C^{\infty}$. Conversely, if $f \in C^{\infty}$, each $f_{n}=0$ so $F(z)$ vanishes identically. Finally, given $z_{0}$ in $\mathcal{D}$, we can find, arguing as in the proof of Theorem 1 , a circle $\left|z-z_{0}\right| \leqq r, r>0$, such that for any $f$ in $C,\left|z-z_{0}\right| \leqq r,|F(z)| \leqq K\|f\|$, where $K$ depends only on $r$. Hence convergence in norm in $C$ implies uniform convergence on compact sets in $D$.

COROLLARY. If $h$ is any generator of $C$, then $H(z)=\sum_{0}^{\infty} h_{n} z^{n} \neq 0$ in $D$.

Proof. Since $h$ is a generator of $C$, there exist polynomials $P_{n}$ with $P_{n}(T) h$ converging to $x$. Since the constant 1 is the image of $x$ in $\mathcal{F}$, Theorem 2 gives that $P_{n}(z) H(z)$ converges to 1 at every $z$ in $\mathscr{D}$. Hence $H(z)$ must $\neq 00$ for all $z$.

Definition. $\mathfrak{A}_{T}$ denotes the ring of all bounded operators $U$ on $C$ with $U T=T U$. 
Theorem 3. Suppose $C^{\infty}=(0)$. Then each $U \neq 0$ in $\mathscr{A}_{T}$ is represented on $\mathcal{F}$ as multiplication by an analytic function $u(z)$ in $\mathcal{F}, u(z) \neq 0$, and so $\mathfrak{A}_{T}$ is isomorphic to a ring of functions analytic in $D$. The null-space of each $U$ in $\mathfrak{Q}_{T}$ consists of 0 alone. In particular, no nontrivial projection $E, E^{2}=E, E \neq I$, and $E \neq 0$, can belong to $\mathfrak{A}_{r}$.

Proof. If $U$ is in $\mathfrak{A}_{T}, U \neq 0$, then $U x \neq 0$ and so, since by hypothesis $C^{\infty}=(0)$, we have a function $u(z)$ in $\mathcal{F}$, not identically zero, corresponding to $U x$. Let now $f$ be arbitrary in $C$ and choose $P_{n}$ with $P_{n}(T) x$ converging to $f$. Then $P_{n}(T) U x=U P_{n}(T) x$ converges to $U f$. But $P_{n}(T) U x$ corresponds in $\mathcal{F}$ to $P_{n}(z) u(z)$. It follows by Theorem 2 that for each $z$ in $\mathscr{D}, P_{n}(z) u(z)$ converges to the value at $z$ of the function in $\mathcal{F}$ which represents $U f$. Finally, in the same way, we know that $P_{n}(z)$ converges to $F(z)$ for all $z$ in $D$, where $F(z)$ is the function in $\mathcal{F}$ corresponding to $f$.

Thus $U f$ maps into $u(z) F(z)$ in $\mathcal{F}$ and so $U$ is represented on $\mathcal{F}$ as multiplication by $u(z)$. If now $U f=0$, for some $f$ in $C, u(z) F(z)=0$ for all $z$ in $D$. Since $u(z)$ is analytic in and not identically $0, u(z)$ has only isolated zeros in $\mathcal{D}$ and so $F(z) \equiv 0$ in $\mathcal{D}$, whence $f=0$, since $C^{\infty}=(0)$. Thus the null-space of $U$ consists of 0 alone. Finally, let $E$ be in $\mathfrak{A}_{T}$ with $E^{2}=E$. If $E \neq I, y=E x-x \neq 0$ for some $x$. Then $E y=0$ and so by the preceding, $E=0$.

2. Let $\tau$ be a normal operator on Hilbert space $H$ and let $C$ be any closed invariant subspace. It is not always true that the operator $T$ obtained by restricting $\tau$ to $C$ is normal. The author showed in [2, Theorem 7] that this is true provided that the spectrum of $\tau$ lacks interior and does not separate the plane. We proceed now to consider the following case: the spectrum of $\tau$ lacks interior and separates the plane into exactly two components.

Let $T$ denote the restriction of $\tau$ to $C$ and let $C$ have a single generator. We do not demand that $C^{\infty} \neq C$. Then under the preceding assumption on the spectrum of $\tau$ we have:

THEOREM 4. Let $C^{0}$ denote the orthogonal complement of $C^{\infty}$ in $C$. Then both $C^{0}$ and $C^{\infty}$ are invariant under $T$. Restricted to the Hilbert space $C^{\infty}, T$ is a normal operator, while restricted to $C^{0}, T$ can be isomorphically represented as multiplication by $z$ on a certain Hilbert space F of analytic functions.

Proof. $C^{\infty}$ is invariant under $\tau$ and $\tau^{-1}$. Let now $\tau=\int \lambda d E_{\lambda}$ be the representation of $\tau$ on the whole Hilbert space $H$. Choose any $\psi$ in $H$ with $\left(\psi, C^{\infty}\right)=0$ and any $f$ in $C^{\infty}$. Then $\left(\psi, \tau^{n} f\right)=0$ for $n=0, \pm 1$, $\pm 2, \cdots$. Hence $\int d\left(E_{\lambda} f, \psi\right) /(\lambda-z)=0$ for all $z$ not in the spectrum of $\tau$. Since the spectrum lacks interior, it follows that $d\left(E_{\lambda} f, \psi\right)=0$ as a 
measure. Thus $\left(\psi, \tau^{*} f\right)=\int \bar{\lambda} d\left(E_{\lambda} f, \psi\right)=0$. Hence $\tau^{*} f \in C^{\infty}$ whenever $f \in C^{\infty}$, and so $\tau$, and hence $T$, is normal, restricted to $C^{\infty}$. Let now $g$ belong to $C^{0}$ and $f$ be in $C^{\infty}$. Then $(T g, f)=\left(g, \tau^{*} f\right)=0$, since $\tau^{*} f \in C^{\infty}$ and $g$ is orthogonal to $C^{\infty}$. Thus $C^{0}$ is invariant under $T$.

Let now $x$ be the generator of $C$ and let $x_{0}$ and $x_{\infty}$ be the components of $x$ in $C^{0}$ and $C^{\infty}$ respectively. We claim that $x_{0}$ is a generator for $C^{0}$. For if $g \in C^{0},\left(g, T^{n} x_{0}\right)=0$, then $\left(T^{n} x, g\right)=0$, and so $g=0$.

Suppose now $g \in C^{0}, \tau^{-n} g \in C^{0}$ for all $n \geqq 0$. Then $\tau^{-n} g \in C, n \geqq 0$, and so $g \in C^{\infty}$. Hence $g=0$. Thus Theorem 2 gives us the representation of $C^{0}$ and $T$, as asserted.

Theorem 4 includes in particular the case when $\tau$ is unitary. Here we can give a simple concrete representation for $\mathcal{F}$. Let $H^{2}$ denote the space of functions analytic in $|z|<1$ and with $\sup _{r<1} \int_{-\pi}^{\pi}\left|F\left(r e^{i \theta}\right)\right|^{2} d \theta<\infty$. It is well known that a Taylor series $\sum_{0}^{\infty} c_{n} z^{n}$ belongs to $H^{2}$ if and only if $\sum_{0}^{\infty}\left|c_{n}\right|^{2}<\infty$. We now have:

TheOREM 5. Let $\tau$ be unitary. Then the space $\mathcal{F}$ of Theorem 4 can be taken to be $H^{2}$, unless $C^{\infty}=C$, in which case $\mathcal{F}=(0)$.

In order to prove Theorem 5 , it suffices to exhibit an orthonormal basis $\phi_{k}$ in $C^{0}$ such that $\phi_{k}=\tau^{k} \phi_{0}, k=0,1,2, \cdots$. For then every $f$ in $C^{0}$ can be written in the form $f=\sum_{0}^{\infty} c_{n} T^{n} \phi_{0}$, where $\sum_{0}^{\infty}\left|c_{n}\right|^{2}<\infty$, and conversely, given $\left\{c_{n}\right\}_{0}^{\infty}$ with $\sum_{0}^{\infty}\left|c_{n}\right|^{2}<\infty$, the vector $\sum_{0}^{\infty} c_{n} T^{n} \phi_{0} \in C^{0}$. Thus $C^{0}$ is isomorphic with $H^{2}$ and clearly this isomorphism carries $T$ into multiplication by $z$, and so the theorem is proved.

The problem of constructing such a basis arises in the study of the representation of "stationary sequences" in Hilbert space. A "stationary sequence" is a sequence of vectors $\left\{x_{n}\right\}_{-\infty}^{\infty}$ such that $\left(x_{n}, x_{m}\right)$ is a function of $n-m$. It is easily seen that a given sequence $\left\{x_{n}\right\}_{-\infty}^{\infty}$ is stationary if and only if there exists a unitary operator $\tau$ with $x_{n}=\tau^{n} x_{0}, n=0, \pm 1, \pm 2, \cdots$. In his paper, Stationary sequences in Hilbert space [3] (cf. also [4]) Kolmogoroff states, without a complete proof, a result of Wold on stationary sequences which yields a basis $\left\{\tau^{k} \phi\right\}_{0}^{\infty}$ for $C^{0}$. We now give a complete argument:

Proof. If $C^{\infty}=C$, then $C^{0}=(0)$ and so $\mathcal{f}=(0)$. Otherwise, $\tau^{-1} x \notin C$. Hence there exist vectors $\chi$ and $\psi, \chi \perp C$ and $\psi$ in $C$, with $\chi \neq 0$, such that $\tau^{-1} x=\chi+\psi$. Setting $\phi=\tau \chi$, we get that $\phi \in C$ and that the vectors $(1 /\|\phi\|) T^{n} \phi, n=0,1, \cdots$, form an orthonormal set. Let $C_{\phi}$ denote the subspace of $C$ spanned by the $T^{n} \phi . C_{\phi} \subseteq C^{0}$, since if $y \in C^{\infty},\left(y, T^{n} \phi\right)=\left(\tau^{-n-1} y, \chi\right)=0$ because $\tau^{-n-1} y \in C$ and $\chi \perp C$, and so $C_{\phi} \perp C^{\infty}$. We assert that $C_{\phi}=C^{0}$, and for this it suffices to show that the orthogonal complement of $C_{\phi} \subseteq C^{\infty}$. 
Let now $E$ be the operator which projects $C$ on the orthogonal complement of $C_{\phi}$. Then $E T=T E$. Also since $\tau^{-1} x=\chi+\psi, E x$ $=E(T \psi)=T(E \psi) \in C^{1}$. Choose now $P_{n}$ with $P_{n}(T) x$ converging to $\psi$. Then $E \psi=\lim _{n=\infty} E P_{n}(T) x=\lim _{n=\infty} P_{n}(T) E x \in C^{1}$ and so $E x$ $=T(E \psi) \in C^{2}$. Repeating the argument, we see that $E \psi \in C^{2}$, hence that $E x \in C^{3}$, and so on. Thus $E x \in C^{\infty}$ and so $E(C) \subseteq C^{\infty}$. But $E(C)$ is the orthogonal complement of $C_{\phi}$, and so we see that $C_{\phi}=C^{0}$, as claimed. By the above, this proves Theorem 5 .

CoRollary. If $\tau$ is unitary and $C^{\infty}=(0)$, then $\mathfrak{P}_{T}$ is isomorphic to the ring of all functions analytic and bounded in $|z|<1$.

Proof. The assertion follows from Theorems 3 and 5.

Kolmogoroff in [3] gave a necessary and sufficient condition that $C^{\infty}=(0)$. In terms of our definitions his result is as follows:

THEOREM (Kolmogoroff): Let $\tau=\int_{-\pi}^{\pi} e^{i t} d E_{t}$ be the spectral representation of the unitary operator $\tau$ on the whole Hilbert space. If $x$ is the generator of $C$, then $C^{\infty}=(0)$ if and only if the measure $d\left(E_{t} x, x\right)$, defined on $(-\pi, \pi)$, has a positive density $p(t)$ such that $d\left(E_{t} x, x\right)=p(t) d t$ and $\log p(t)$ is summable.

The operator $T_{0}$ of multiplication by $z$ in $H^{2}$, which, as we have seen, arises in the restriction of unitary operators, has been extensively studied by Beurling in [5]. He determined all closed invariant subspaces of $T_{0}$ and gave a necessary and sufficient condition on a vector $h$ in $H^{2}$ in order that $h$ be a generator of $H^{2}$ under $T_{0}$. The necessary condition of the corollary to our Theorem 2 is not sufficient.

The systematic study of restrictions of normal operators was begun by Halmos in [6].

\section{REFERENCES}

1. T. Hall, Thesis, Uppsala, 1950.

2. J. Wermer, On invariant subspaces of normal operators, Proc. Amer. Math. Soc. vol. 3 (1952) pp. 270-277.

3. A. N. Kolmogoroff, Stationary sequences in Hilbert's space, Bolletin Moskovskogo Gosudarstvenogo Universiteta Matematika vol. 2 (1941) (Mathematical Reviews vol. 5 (1944) p. 101).

4. - Interpolation und Extrapolation von stationären zufälligen Folgen, Bull. Acad. Sci. URSS. Ser. Math. Izvestia Akad. Nauk. SSSR vol. 5 (1941) pp. 3-14.

5. A. Beurling, On two problems concerning linear transformations in Hilbert space, Acta Math. vol. 81.

6. P. R. Halmos, Normal dilations and extensions of operalors, Summa Brasiliensis Math. Fasc. 9, vol. II, 1950.

Yale University 